\title{
An Overview on Low-Level Laser Therapy (LLLT) \& Cooling Laser Therapy (C.L.T.) in Medical Engineering
}

\author{
Majid Monajjemi 1,*(D), Afshar Alihosseini ${ }^{1}$, Fatma Kandemirli ${ }^{2}$ (D) , Fatemeh Mollaamin ${ }^{2(\mathbb{D})}$ \\ 1 Department of Chemical engineering, Central Tehran Branch, Islamic Azad University, Tehran, Iran \\ 2 Department of Biomedical Engineering, Faculty of Engineering and Architecture, Kastamonu University, Kastamonu, \\ Turkey \\ * Correspondence: m_monajjemi@ srbiau.ac.ir; maj.monajjemi@iauctb.ac.ir (M.M.);
}

Scopus Author ID 670181068

Received: 4.07.2021; Revised: 1.10.2021; Accepted: 5.10.2021; Published: 8.11.2021

\begin{abstract}
Some essential methods for overcoming the skin problem during laser therapy are Low-Level Laser Therapy and Cooling Laser Therapy in cooling the most superficial layers of the skin. Although melanin absorption will result in heat production during laser exposure, cooling the epidermis can prevent its temperature elevation from exceeding the threshold for thermal injury. L.D.C. is a technique for laser cooling of small particles. Low-Level Laser Therapy (LLLT), consisting of Low-intensity radiation in specific wavelengths, has been discussed to trigger cellular proliferation and differentiation through molecular mechanic mechanisms. This work studied the propagation of the laser beam into biological tissue such as the brain and skin by the Monte Carlo method. What is generally accepted is that the energy density required is very small, and the transmit power is less than 0.6 Watts
\end{abstract}

Keywords: Low-Level Laser Therapy; Cooling Laser Therapy; medical engineering, photobiological \& photochemical.

(C) 2021 by the authors. This article is an open-access article distributed under the terms and conditions of the Creative Commons Attribution (CC BY) license (https://creativecommons.org/licenses/by/4.0/).

\section{Introduction}

Laser cooling consists of several techniques in which atomic and molecular samples are cooled down to near -273 centigrade. The laser cooling technique's root is based on the fact that when an atom absorbs and reflexes a photon, its momentum would be changed for those particles. Also, their thermodynamic temperatures are proportional to the variance in their velocities [1-5]. This means more homogeneous velocities between two particles related to a lower temperature. There are several various methods for laser cooling such as Doppler cooling [5-14], Sisyphus cooling, Resolved sideband cooling, Raman sideband cooling, Velocity selective coherent population trapping (VSCPT), Gray molasses, Cavity mediated cooling, Use of a Zeeman slower, Electromagnetically induced and transparency (EIT) cooling, and AntiStokes cooling in solids. Laser cooling methods; make the atomic spectroscopy via adding mechanical effect to create the uniform velocity distribution of the particles. Consequently, the effect will reduce the thermal movements in atoms with external force via a laser beam. Generally, this kind of cooling is produced for reducing the temperature of a di-atomic gas and its velocity in any beams. Light affects atomic motion when the atoms absorb or emit photons. The momentum of Photons is $p=h / \lambda$, where $\lambda$ is the light's wavelength, and when an atom absorbs or emits a photon, the atom's momentum has to be changed through a value equal to the photon momentum $[2,5]$. By any absorption or emission, by a tiny kick to atoms, their velocity will be changed, and for all atoms, this changing is about a few millimeters, while for 
the atom gases, the changing of speed is about several hundred up to a thousand meters per second. Anyway, repeated absorption and emission of photons might be having a huge effect on even hot atomic gases or beams. In the 1970s, researchers investigated techniques for cooling atoms to very low temperatures using laser light (Scheme 1).

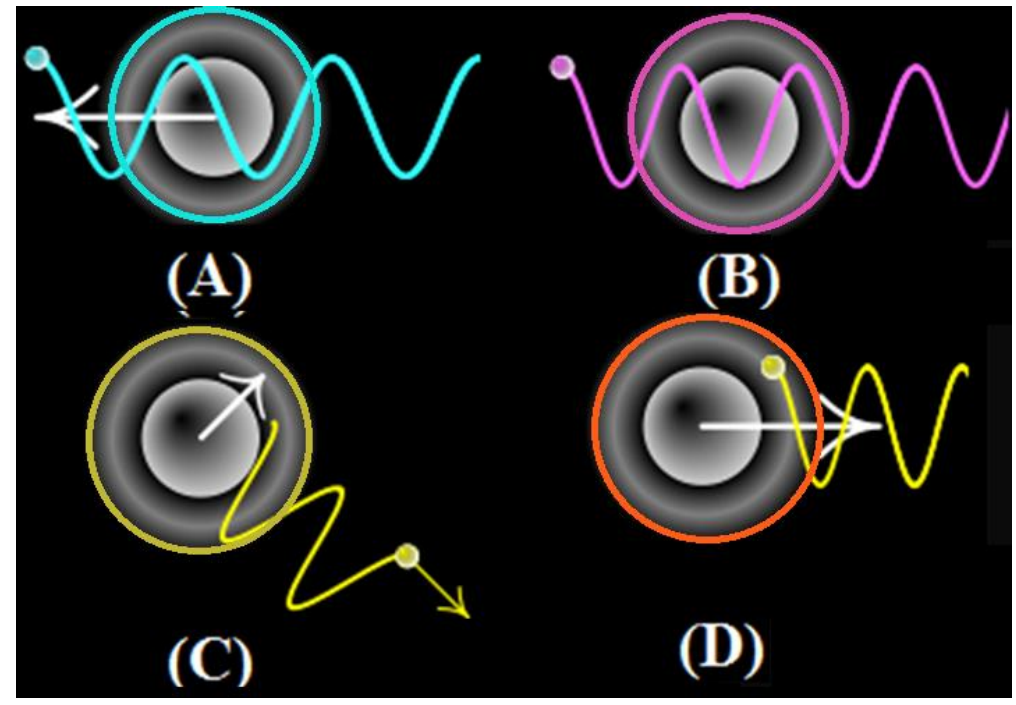

Scheme 1. (A) The atom moving away from the laser and does not absorb the photon (red-shifted); (B) The stationary atom that can not absorb the photon (neither red- nor blue-shifted); (C) The atom re-emits a photon including no changing in momentum with several absorption-emission; (D) An atom moving towards the laser sees it blue-shifted and absorbs the photon, slowing the atom

\subsection{Laser Doppler Cooling (L.D.C.).}

L.D.C. is a technique for laser cooling of small particles. This method is based on a subsequent spontaneous photons emission where lead to light forces, and these forces become velocitydependent through the Doppler effect [6-13]: an absorption resonance of an atom or ion is shifted, e.g., towards lower frequencies when the particle is moving towards the light source. For example, a beam of atoms in a vacuum cell can be cooled with a counter-propagating single beam, the frequency of which is first chosen to be higher than the atomic resonance; therefore that only the fastest atoms can absorb photons. During photon absorbance, the atoms are in an excited state and return to the original state by spontaneously emitting a photon. These photons are radiated in several orientations, so the results of their kicks average to zero [7,8]. For single atoms, another similarly-tuned beam can be added, targeted in the opposite form, for cooling those atoms moving in the opposite orientation. Then, no matter the orientation of an atom's speed, one or two beams oppose the speed and slow the atom (Scheme 2). Consequently, the beam frequency of the laser is decreased, so that very slower atoms participate in the interaction, and then, all atoms have a largely decreased velocity. These liaise to a down temperature, supposing that thermal equilibrium can be reestablished. Further sweeping changes of the laser frequency are several atomic resonances through a spatially varying magnetic field.

Doppler cooling (DC effect) might also be applied in optical molasses. The minimum temperature accessible with DC effect is named the Doppler limit. In some items, the Doppler limit has been interpreted as Sisyphus cooling [12, 13]. In the 1970s, researchers discovered the use of lasers for providing cool atoms to temperatures above absolute zero[11,14]. A few papers from that era, all published in Physical Review Letters, highlight some important steps in developing the technology. In 1978, researchers cooled ions somewhat below 50 Kelvin; ten 
years later, neutral atoms had gotten a million times colder to 40 micro kelvins. In addition, the fundamental explored based on the force of laser light applied to atoms to slow them down [1315], which is known as a new quantum form of matter called a Bose-Einstein condensate and modern atomic clocks and at least two Nobel prizes.

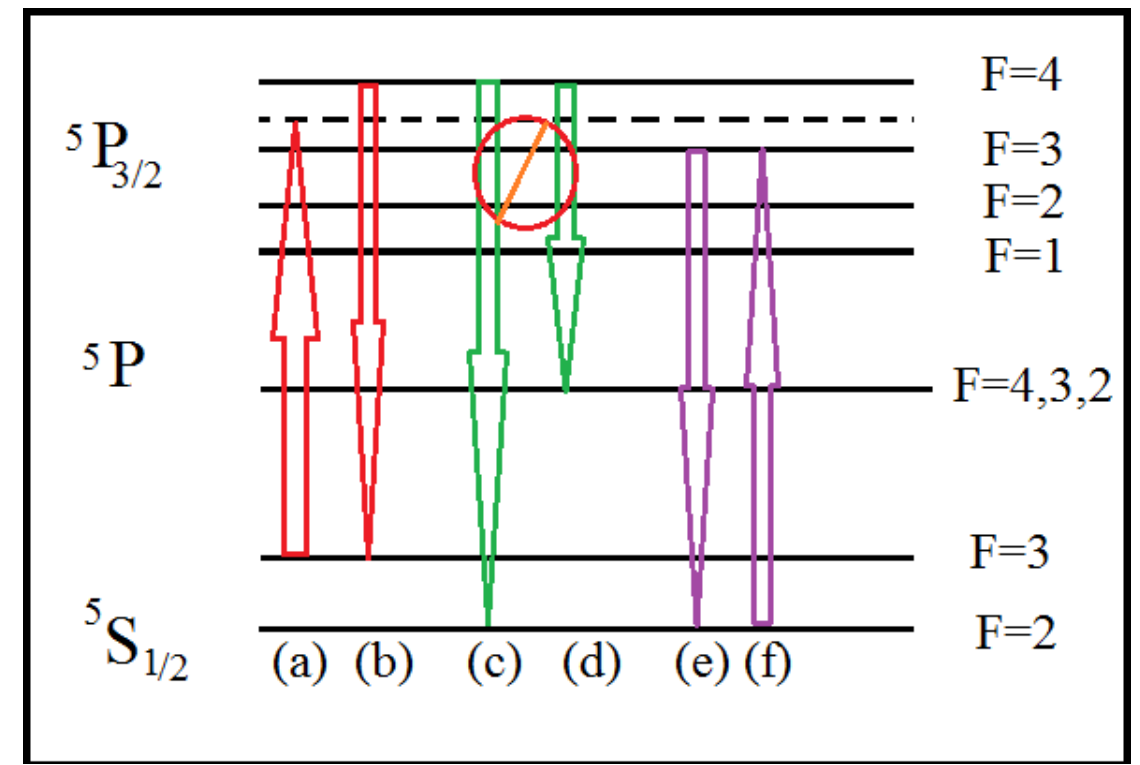

Scheme 2. (a) \& (b) exhibit the absorption and spontaneous emission cycle, (c) \& (d) are forbidden transitions, (e) exhibits that if a cooling laser excites an atom to the $\mathrm{F}=3$ state, it is allowed to decay to the "dark" lower hyperfine, $\mathrm{F}=2$ state, which would stop the cooling process and (f) if it were not for the repumper laser (f).

The fundamental reason to cool atoms-that is, reduce the velocity of atomics motionwas to allow more accurate measurement of atomic spectra. In 1978 Dave Winel and his team pursued theoretical approaches and managed to laser cool magnesium ions [14-16]. They limited the ions in an electromagnetic lasso and hit them with a laser beam to a frequency close to "resonance" frequency of those ions. At relaxation time, the ions absorb several photons at these resonance frequencies, and they move toward the beam, but its lower frequency appears. These photons slow down the ions until the cooling effect is balanced via the small heating. In other words, it causes emitting or absorbing a photon in any orientation that would ultimately limit the cooling possible with this (so-called Doppler cooling technique) if the laser frequencies are tuned to be slightly lower than those atoms moving against the laser beam [16$18]$.

\subsection{Sisyphus cooling laser.}

Sisyphus cooling is a kind of laser cooling of atoms used to reach temperatures below the Doppler condition. This approach was first investigated by Claude Cohen-Tannoudji (Nobel Prize in Physics) in 1989 via Na atom that cooled below the Doppler position in optical molasses. Sisyphus laser can be achieved via two orthogonal polarization laser lights inside an atom. Consequently, atoms move through the different potentials along with the standing kinetic energies to a higher potential, at which point optical pumping moves them back to a lower energy state, thus lowering the atom's total energy. This description of Sisyphus cooling is largely based on Foot's description (Scheme 3). 


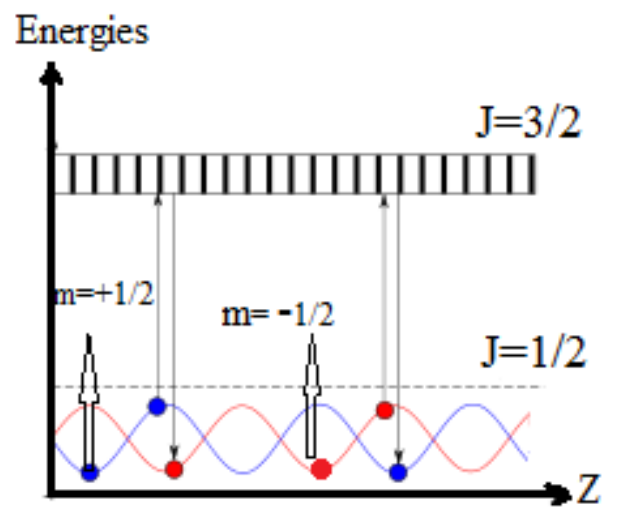

Scheme 3. Sisyphus cooling: The atoms are running against the potential energy, become excited into a higher band, fall back into a low-energy state.

The counter-propagation or ( lin perp lin) configuration, produces a polarization wave between two states (phase difference $=\pi / 2$ ) of $\sigma^{ \pm}$the gradient that occurs over a length scale of $\frac{\lambda}{2}$, and then repeats, mirrored about the $y-z$ plane. For an atom between ground state $\left(J_{1}=1 / 2\right)$ and excited state $\left(\mathrm{J}_{2}=3 / 2\right)$, the $\mathrm{M}_{\mathrm{J}}$ sublevels are $\left(\mathrm{M}_{\mathrm{J}}= \pm 1 / 2\right)$ and $\left(\mathrm{M}_{\mathrm{J}}= \pm \pm \frac{1}{2},=3 / 2\right)$, for grand and excited states, respectively. In the field-free case, all of these energies for each $\mathrm{J}$ amount are degenerate, but in the presence of a circularly polarized light field, the Autler-Townes effect lifts this degeneracy. This polarization applies a spatially-dependent slowing force to the atom. Laser cooling is closely connected with the trapping of atoms due to their slowed-downs before they can be held in a trap and must often be trapped to observe laser cooling. Such phenomenon consists of cold, trapped ions arranging themselves into a crystal (Due to the electrical repulsion among the ions).

\subsection{Low-Level Laser Therapy (LLLT).}

The photobiological-photochemical phenomena arising from laser effects in human tissues have the same photosynthesis mechanism in plants. Theoretically, the radiation red or near-infrared spectrum makes stimulation of mitochondria [19]. Characteristic examples of chromophores are observed in chlorophyll, hemoglobin, Cytochrome Oxidase C (COX), myoglobin, flavo-proteins, and porphyrins [20]. The photoreceptor molecules of mitochondria, on which laser radiation seems to act as a photo-stimulant, can trigger a series of photochemical reactions, which in turn can cause changes in cellular metabolisms, such as protein signaling. The function photoreceptors are COX, the last enzyme in the electron transport chain of mitochondria. Those photoreceptors are NADPH oxidase, Nitric Oxide (NO), cytochrome family "aa and aa3 quinol oxidase", while the key photoreceptors are the molecule of COX. LLLT mechanism in respiratory re-start via NO release through $\mathrm{Fe}$ ions and $\mathrm{Cu}-\mathrm{COX}, \mathrm{O}_{2}$ recapture, and ATP production increase. It must be noted that the release of NO under the influence of low-power laser radiation is affected by other cell storages (hemoglobin and myoglobin proteins) which capture NO [21]. In addition, LLLT, induces the synthesis of transcription agents. This system is arranged through increasing ROS production, which causes the increased output of hydroperoxide anion and finally increased concentration of hydrogen peroxide [22]. The initial reactions that have been accomplished in the mitochondria can be followed via a collection of chains that appear in the cell resulting in cellular homeostasis. So, change is observed in the $\mathrm{pH}$, in the concentration of intracellular $\mathrm{Ca} 2+$, in the ATP and the cAMP, as well as in the redox potential. The situation described above, which has been 
supposed to be a possible interpretation of the effect of radiation on tissues, is quite complicated, promoting tissue reproduction, preventing cell death, analgesic action, and relieving neurogenic pain (laser acupuncture), anti-inflammatory activity, etc. The synthesis of DNA and RNA and the increase of cell proliferation is induced by the increase of the intracellular $\mathrm{Ca} 2+$ concentration provoked by LLLT activity on the $\mathrm{Ca}^{2+}$ channels [23].

Notwithstanding what has been illustrated above regarding the mechanisms by which LLLT exerts its bio-stimulatory action, the real mechanism that causes analgesia has not been identified. It has been believed that the laser light selectively stimulates the free nerve endings, which are placed in the superficial sheets of the skin.

\section{Materials and Methods}

\subsection{Simulation model.}

COMSOL Multiphasic Simulation Software has been used to model the system, which consists of thermal conduction and formulated based on Fourier's transformation. The Heat Transfer Modulation has been used for several tissues, such as the brain. The Brain material with tumors can be defined by densities, conductivities, specific heating, diffusivities, relative permittivity, relative permeability. The COMSOL Multiphasic model is converted into an application with its interface using the tools provided with the Application Builder desktop environment. A cylinder $3 * 3 \mathrm{~cm}$ of radius and thickness brain tissue has been provided and heated up to 15 seconds by a $10 \mathrm{~W}$ laser (initial temperature was kept $293.15 \mathrm{~K}$ ). The laser beam is simulated as a heat source with a Gaussian profile (Figure 1). This system uses the built-in Gaussian Pulse functions, which enforces that the integral under the curve equals unity.

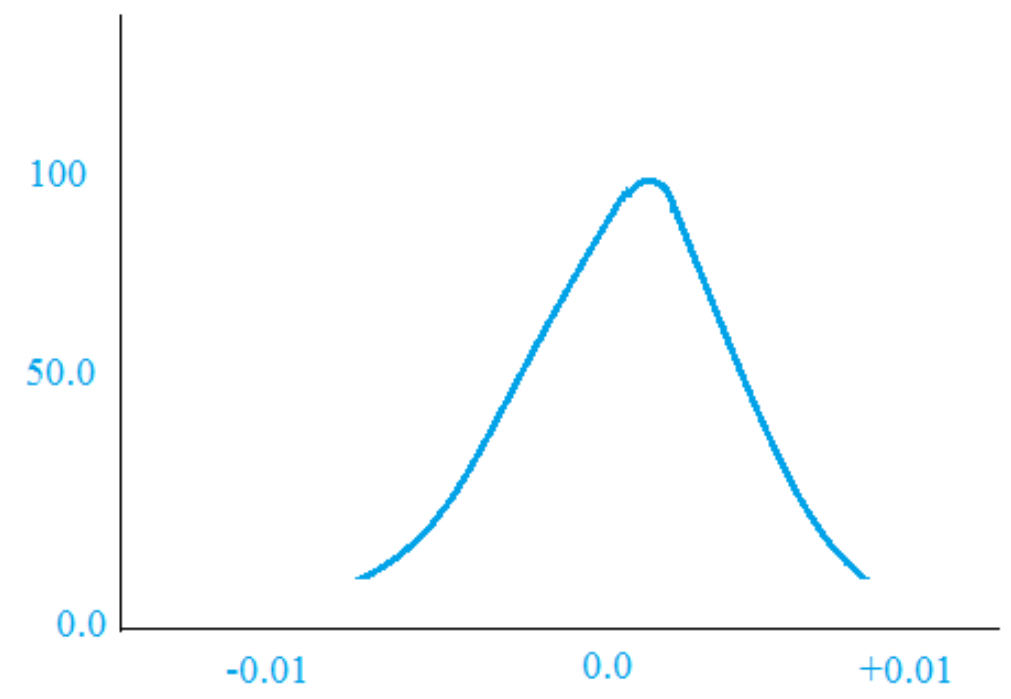

Figure 1. The Gaussian Pulse with location 0 and Standard deviation.

The LITT of Brain Tumors can be modeled by the bio-heat equation in a 3D geometric, using the bioheat transfer application mode with time-dependent in COMSOL 5.1. The heat equation was described as $\rho \boldsymbol{C}_{\boldsymbol{p}} \frac{\boldsymbol{\partial} \boldsymbol{T}}{\boldsymbol{\partial} \boldsymbol{t}}+\boldsymbol{\rho} \boldsymbol{C}_{\boldsymbol{p}} \boldsymbol{u}$. $\boldsymbol{\nabla T}+\boldsymbol{\nabla} \boldsymbol{q}=\boldsymbol{Q}+\boldsymbol{Q}_{\boldsymbol{b i o}}$ and $\mathbf{q}=-\mathbf{k} \boldsymbol{\nabla}$ where $\mathrm{T}$ is the temperature $(\mathrm{K})$, and $\boldsymbol{C}_{\boldsymbol{p}}$ is the heat capacity $\left.\mathrm{J} /\left(\mathrm{kg}^{*} \mathrm{~K}\right)\right), \boldsymbol{\rho}$ is the density of the brain tissue $\left(\mathrm{kg} / \mathrm{m}^{\wedge} 3\right)$, and $\mathrm{k}$ is the thermal conductivity of brain tissue $\left(\mathrm{W} /\left(\mathrm{m}^{*} \mathrm{~K}\right), \boldsymbol{Q}\right.$ is the heat source, is the perfusion and metabolic heat source. Here $\boldsymbol{Q}_{\boldsymbol{b i o}}$ is the perfusion and metabolic heat source and define as $\boldsymbol{Q}_{\boldsymbol{b i o}}=\boldsymbol{\rho}_{\boldsymbol{b}} \boldsymbol{C}_{\boldsymbol{b}} \boldsymbol{\omega}_{\boldsymbol{b}}\left(\boldsymbol{T}_{\boldsymbol{b}}-\boldsymbol{T}\right)+\boldsymbol{Q}_{\boldsymbol{m} \boldsymbol{e t}}$, where $\boldsymbol{\rho}_{\boldsymbol{b}}$ is the blood density, $\boldsymbol{C}_{\boldsymbol{b}}$ is specific 
blood heat, $\boldsymbol{Q}_{\boldsymbol{m} e t}$ is the metabolic heat source, $\boldsymbol{\omega}_{\boldsymbol{b}}$ is blood perfusion rate, $\boldsymbol{T}_{\boldsymbol{b}}$ is the blood flow rate, $\mathrm{T}$ is time (s).

\subsection{Monte Carlo simulation.}

The main base of simulation in biological tissues is the Monte Carlo method [24]. The Monte Carlo method is applied to simulate light propagation in biological tissue based on the photon propagation in absorption and scattering medium [25-40]. The effects of data can be investigated using Monte-Carlo simulations. In the simple case, the photon is put into an autonomous media, and its movement can be registered up to its scattering. Although the results are extremely precise, this method requires repeated calculations several times for achieving favorable measuring. In other words, stopping for each photon that will scatter until an absorption process finishes it must be consecrated. For this purpose, these photons are given an "initial weight", and gradually, these weights decrease with each move at each scattering position. This investigates the performance of the statistical method, which avoids the computational process of the photons moving several steps to make it just end in absorption procedure [41-47].

\subsection{Mechanism of active cooling.}

Several methods have been investigated for cooling human skins and other tissue via laser therapy. All methods apply a precooled medium that can be solid, liquid, or gas, brought into contact with the skin surface. A deeper skin segment is cooled by heat diffusion toward the cooled section, with subsequent transfer to the cooling medium. The heat transfer rate across the interface among the skin layers and cooling average depends primarily on the temperature difference between the 2 adjacent layers and other parameters specific to each cooling way. Under most situations, the heat transfer rate is roughly proportional to the temperature difference among the materials. So, cooling efficiency using any method could be adequately characterized through the proportionality constant, termed heat transfer coefficient, and other physical parameters. The rate of heat extraction from human skins is restricted by the stratum corneum, which has a very low thermal conductivity due to its low hydration and low viscous topical hydrating gel can increase stratum corneum conductivities.

Such "passive" cooling reduces heat buildup near the skin surfaces after laser exposure but cannot produce the spatial selectivity required for enhanced epidermal protection during laser exposure. The hydrating gel can be applied with active cooling systems. Contact cooling (CC) of skin is gained through heat conduction into an adjacent precooled solid body. Therapeutic laser exposure is delivered through the sheet, which is pressed against the patient's skin. Contact cooling appears when a highly conductive material, such as sapphire is used for the cooling sheet. When CC is used clinically, a layer including bubbles, hair, fatty acids, water, thick (>25/Lm) hydrating gel may prevent direct contact among the skin surfaces and cooling plates.

Using a sapphire plate cooled to $-5^{\circ} \mathrm{C}$, the cooling rate is extremely slow compared to that of commercial cryogen spray cooling (CSC). Such sheet temperature state a considerable risk of irreversible skin injuries due to the lack of automated control over the cooling time. In the laboratory, the heat transfer coefficient among sapphire sheets (at $5^{\circ}$ to $10^{\circ} \mathrm{C}$ ) and an epoxy phantom were found a variation of one thousand to ten thousand $\mathrm{W} / \mathrm{m}^{2} k$. Although, by increasing the light dosage of epidermal thermal damage, it is critically important to have 
control of the induced epidermal cooling. Similar concerns may also be raised with respect to transient freezing of the superficial epidermal sheets using such an aggressive cooling regime.

\subsection{Cryogen spray cooling (CSC).}

The major cooling approach of CSC is rapid evaporation of cryogen, which extracts latent heat of vaporization from the surrounding environment (Figure 2). Several cryogenic compounds are currently used in dermatology, such as TFE (C2H2F4, known as R134a). It has a boiling point at $-25.9^{\circ} \mathrm{C}$, which results in a much higher evaporation rate and, therefore, faster heat extraction when compared to chilled water sprays. On release from a pressurized container, liquid cryogen is atomized into a fine spray and directed toward the skin surface a few centimeters away[48-67].

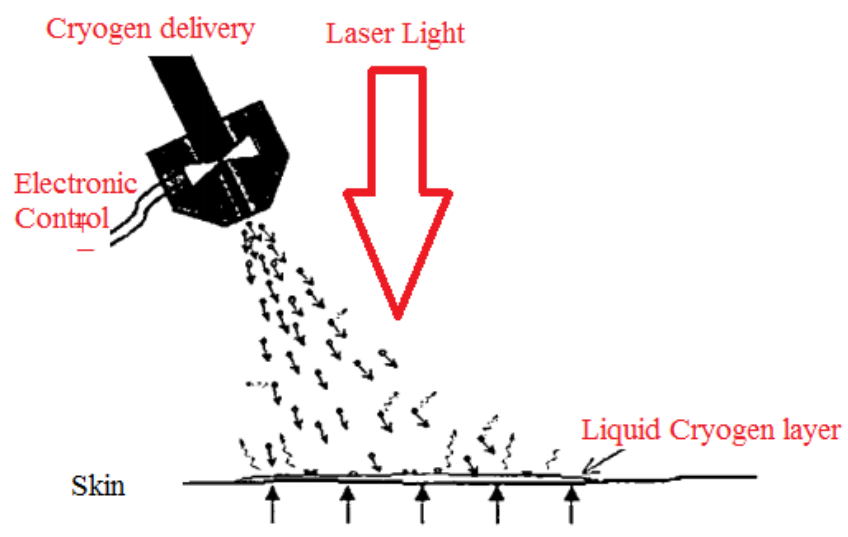

Figure 2. CSC mechanism for human skin.

When impinging on the skin surface, droplet temperature is typically between $-45^{\circ}$ and $-65^{\circ} \mathrm{C}$, depending on the distance between the skin surface and atomizer nozzle design. The CSC provides a rapid, large, and spatially selective epidermal temperature reduction.

\section{Results and Discussion}

For this simulation, a segmented tissue of the human skin, including stratum-corneum, epidermis, dermis with a thickness between $(0.05-0.15 \mathrm{~cm})$, can be considered for simulation based on some previous works $[13,14]$. The first simulation from skin consists of skin $0.1 \mathrm{~cm}$; fat and $0.9 \mathrm{~cm}$; muscle. The absorption coefficient and the scattering coefficient are defined as $\mu \mathrm{a}$ and $\mu \mathrm{s}$, respectively, probability density functions. Their inverse can be explained as the average mean distance for absorption and scattering. The total attenuation coefficient $\mu_{t}$ is the sum of the absorption coefficient $\mu_{a}$ and scattering coefficient $\mu_{s}$, which characterize the interaction of the photon average per unit of path length. The simulated characterize are listed in Table 1.

Table 1. The optical parameters of the tissues.

\begin{tabular}{|c|c|c|c|c|c|}
\hline & Tissue & Wave length (nm) & $\mathbf{n}$ & $\mu a \mathrm{~cm}^{-1}$ & $\mu_{s} \mathrm{~cm}^{-1}$ \\
\hline \multirow{3}{*}{ connective } & bone & 690 & \multirow{3}{*}{1.45} & 0.123 & 223 \\
\hline & lymph tissues & 705 & & 0.143 & 244 \\
\hline & blood & 695 & & 0.155 & 195 \\
\hline \multirow[t]{2}{*}{ epithelial } & Skin & 770 & \multirow[t]{2}{*}{1.42} & 0.198 & 266 \\
\hline & Mucous membrane & 750 & & 0.163 & 298 \\
\hline \multirow[t]{3}{*}{ muscle } & skeletal & 780 & \multirow[t]{3}{*}{1.5} & 0.223 & 342 \\
\hline & smooth & 790 & & 0.323 & 332 \\
\hline & cardiac & 830 & & 0.254 & 234 \\
\hline Nervous & $\mathrm{CNS}$ & 900 & 1.33 & 0.453 & 342 \\
\hline
\end{tabular}




\begin{tabular}{l|l|l|l|l|l}
\multicolumn{2}{c}{ Tissue } & Wave length $(\mathbf{n m})$ & $\mathbf{n}$ & $\boldsymbol{\mu a} \mathbf{c m}^{-1}$ & $\boldsymbol{\mu}_{\boldsymbol{s}} \mathbf{c m}^{-\boldsymbol{I}}$ \\
\hline & PNS & 920 & & 0.354 & 256
\end{tabular}

Skin cooling removes heat from the skin to a contact cooling agent such as gas, liquid, and special solids (Figure 3).

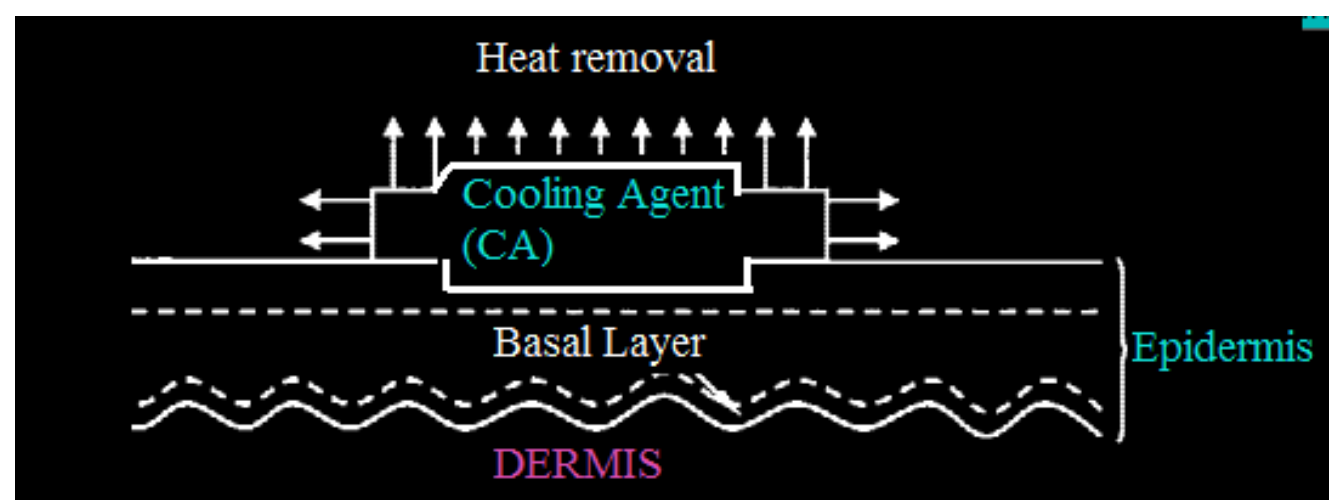

Figure 3. Skin cooling schematic.

The CA might be moved along the skin, as in the case of flowing gas or liquid or a moving solid. Therefore, heat removal from the skin is aided by the mass transfer of the heated CA. For spray cooling, the temperature is smaller than skin temperature. Heat is deleted from the skin into the $\mathrm{CA}$ via conduction and removed from the liquid CA through evaporation. The contact layer is complete during spray duration and forms in time as the liquid boils and evaporates. For solid contact cooling, it should be either active or passive heat removal from the CA. There is no active heat removal from the CA in the passive item, which results in an average temperature rise after contact with skin. As an example, a cold gel is a passive cooler. Hereafter, we consider only active contact and spray in the cooling system.

Theoretical studies predict that efficient cooling appears when the mass flux of sprayed cryogen matches the evaporation rate at the skin surfaces. Heat conducted from deeper skin substrates is converted directly into latent heat of evaporating cryogen droplets. When the spray flux exceeds the sheet's evaporation rate, a liquid cryogen substrate makes up on the skin surface. Under such conditions, heat must be transported from the skin to the evaporation site through the cryogen layer via conduction and forced convection. The additional thermal barrier imposed by this liquid layer reduces the cooling rate. It is notable that larger and faster droplets, produced by less atomizing spray nozzles, can minimize or prevent the formation of the liquid cryogen layer, leading to increased cooling rates. The cryogen spurt duration and the delay between spurt termination and the laser pulse can be controlled electronically, which results in predictable cooling Ivith reproducible spatial selectivity. This approach provides an unparalleled safety margin Ivith respect to preventing undesirable thermal injury by the laser pulse or frostbite attributable to cooling.

\section{Conclusions}

A theoretical investigation of parallel cooling and the conditions required to benefit from this cooling method is also presented. The works conclude that various spray cooling is far more effective and favorable than contact cooling. 


\section{Funding}

This research received no external funding.

\section{Conflicts of Interest}

The authors declare no conflict of interest.

\section{References}

1. Melle, S.; Alavie, A.; Karr, S.; Coroy, T.; Liu, K.; Measures, R. A Bragg grating-tuned fiber laser strain sensor system. IEEE Photon. Technol. Lett. 1993, 5, 263-266.

2. Barot, D.; Wang, G.; Duan, L. High-Resolution Dynamic Strain Sensor Using a Polarization-Maintaining Fiber Bragg Grating. IEEE Photon. Technol. Lett. 2019, 31, 709-712.

3. Pang, Y.; Chen, B.K.; Yu, S.F.; Lingamanaik, S.N. Enhanced laser speckle optical sensor for in situ strain sensing and structural health monitoring. Opt. Lett. 2020, 45, 2331-2334.

4. Pan, Z.; Huang, S.; Su, Y.; Qiao, M.; Zhang, Q. Strain field measurements over $3000{ }^{\circ} \mathrm{C}$ using 3D-Digital image correlation. Opt.Lasers Eng. 2020, 127, 105942.

5. Weisbecker, H.; Cazzolato, B.S.; Wildy, S.; Marburg, S.; Codrington, J.; Kotousov, A.; Kotousov, A. Surface Strain Measurements Using a 3D Scanning Laser Vibrometer. Exp. Mech. 2011, 52, 805-815.

6. Reyes, J.M.; Avitabile, P. Use of 3D Scanning Laser Vibrometer for Full Field Strain Measurements. In Experimental Techniques,Rotating Machinery, and Acoustics; The Society for Experimental Mechanics Series; Springer: Cham, Switzerland 2015, 8, 97-209.

7. Albrecht, H.-E.; Borys, I.M.; Damaschke, D.-I.N.; Tropea, I.C. Basic Measurement Principles. In Laser Doppler and Phase Doppler Measurement Techniques; Springer Science and Business Media LLC: Cham, Switzerland 2003, 9-26.

8. Hercher, M.; Wyntjes, G.; Deweerd, H. Non-Contact Laser Extensometer. In Proceedings of the Industrial Laser Interferometry, Los Angeles, CA, USA, 10 September 1987, 746, 185-193.

9. Bin, L.; Jing-Wen, L.; Chun-Yong, Y. Study on the measurement of in-plane displacement of solid surfaces by laser Doppler velocimetry. Opt. Laser Technol. 1995, 27, 89-93.

10. Gasparetti, M.; Revel, G.M. The influence of operating conditions on the accuracy of in-plane laser Doppler velocimetry measurements. Measurement 1999, 26, 207-220.

11. Zhong, Y.; Zhang, G.; Leng, C.; Zhang, T. A differential laser Doppler system for one-dimensional in-plane motion measurement of MEMS. Measurement 2007, 40, 623-627.

12. Agusanto, K.; Lau, G.-K.; Wu, K.; Liu, T.; Zhu, C.; Yuan, L. Directional-sensitive differential laser Doppler vibrometry for in-plane motion measurement of specular surface. In Proceedings of the International Conference on Experimental Mechanics 2014, Singapore, 15-17 November 2014.

13. Wang, F.; Scholz, A.; Hug, J.; Rembe, C. Laser-Doppler-Dehnungssensor / Laser-Doppler strain gauge. TM Tech. Mess. 2019, 86, 82-86.

14. Rembe, C.; Siegmund, G.; Steger, H.; Wörtge, M. Measuring MEMS in Motion by Laser Doppler Vibrometry. In Optical Science and Engineering; Informa UK Limited: London, UK, 2006, 245-292.

15. Yang, X.; Boggs, J.E.; Extensive ab initio study of the valence and low-lying Rydberg states of BBr including spin-orbit coupling. J Chem Phys. 2006, 124,194307, https://doi.org/10.1063/1.2197830.

16. Li, X.; Zhang, X.; Yan, B. Ab initio study on the low-lying excited states of gas-phase $\mathrm{PH}(+)$ cation including spin-orbit coupling. Spectrochim Acta A Mol Biomol Spectrosc. 2015, 142, 1-7, https://doi.org/10.1016/j.saa.2015.01.070.

17. Gao, Y.; Wan, M. Vibrational branching ratios and radiative lifetimes in the laser cooling of AlBr. Phys Chem Chem Phys. 2017, 19, 5519-5524, https://doi.org/10.1039/c6cp08181a.

18. Wu, DL.; Tan, B.; Wen, YF.; Zeng, XF.; Xie, A.D.; Yan, B, Ab initio MRCI+Q calculations on the low-lying excited states of the MgBr radical including spin-orbit coupling. Spectrochim Acta A Mol Biomol Spectrosc, 2016, 161, 101-8, https://doi.org/10.1016/j.saa.2016.02.027.

19. Wan, M.; Shao, J.; Gao, Y.; Huang, D.; Yang, J.; Cao, Q.; Jin, C.; Wang, F. Laser cooling of $\mathrm{MgCl}$ and $\mathrm{MgBr}$ in theoretical approach , $J$ Chem Phys. 2015, 143, 024302, https://doi.org/10.1063/1.4926389.

20. Tsai, S.R.; Hamblin, M.R, Biological effects and medical applications of infrared radiation. J Photochem Photobiol B. 2017, 170, 197-207. 
21. Shiva S, Gladwin MT (2009) Shining a light on tissue NO stores: near infrared release of NO from nitrite and nitrosylated hemes. J Mol Cell Cardiol, 2009, 46, 1-3.

22. Eslami H, Motahari P, Safari E, Seyyedi M, Evaluation effect of low level Helium-Neon laser and Iranian propolis extract on Collagen Type I gene expression by human gingival fbroblasts: an in vitro study. Laser Ther 2017, 26, 105-112.

23. AlGhamdi KM, Kumar A, Moussa NA, Low-level laser therapy: a useful technique for enhancing the proliferation of various cultured cells. Lasers Med Sci, 2012, 27, 237-249.

24. Metropolis, N.; Ulam, S. The Monte Carlo Method. Journal of the American Statistical Association 1949, 44, 335-341, https://doi.org/10.1080/01621459.1949.10483310.

25. Monajjemi, M. Cell membrane causes the lipid bilayers to behave as variable capacitors: A resonance with self-induction of helical proteins. Biophysical Chemistry 2015, 207, 114-127, https://doi.org/10.1016/j.bpc.2015.10.003.

26. Monajjemi, M. Liquid-phase exfoliation (LPE) of graphite towards graphene: An ab initio study. Journal of Molecular Liquids, 2017, 230, 461-472, https://doi.org/10.1016/j.molliq.2017.01.044.

27. Monajjemi, M.; Naderi, F.; Mollaamin, F.; Khaleghian, M. Drug design outlook by calculation of second virial coefficient as a nano study. Journal of the Mexican Chemical Society 2012, 56, 207-211, https://doi.org/10.29356/jmcs.v56i2.323.

28. Monajjemi, M.; Bagheri, S.; Moosavi, M.S. Symmetry breaking of B2N(-,0,+): An aspect of the electric potential and atomic charges. Molecules 2015, 20, 21636-21657, https://doi.org/10.3390/molecules201219769.

29. Monajjemi, M.; Mohammadian, N.T. S-NICS: An aromaticity criterion for nano molecules. Journal of Computational and Theoretical Nanoscience 2015, 12, 4895-4914, https://doi.org/10.1166/jctn.2015.4458.

30. Monajjemi, M.; Ketabi, S.; Hashemian, Z.M.; Amiri, A. Simulation of DNA bases in water: Comparison of the Monte Carlo algorithm with molecular mechanics force fields. Biochemistry (Moscow) 2006, 71, 1-8, https://doi.org/10.1134/s0006297906130013.

31. Monajjemi, M.; Lee, V.S.; Khaleghian, M.; Honarparvar, B.; Mollaamin, F. Theoretical Description of Electromagnetic Nonbonded Interactions of Radical, Cationic, and Anionic NH2BHNBHNH2 Inside of the B18N18 Nanoring. J. Phys. Chem C 2010, 114, 15315-15330, https://doi.org/10.1021/jp104274z.

32. Monajjemi, M.; Boggs, J.E. A New Generation of BnNn Rings as a Supplement to Boron Nitride Tubes and Cages. J. Phys. Chem. A 2013, 117, 1670-1684, https://dx.doi.org/10.1021/jp312073q.

33. Monajjemi, M. Non bonded interaction between BnNn (stator) and BN B (rotor) systems: A quantum rotation in IR region. Chemical Physics 2013, 425, 29-45, https://doi.org/10.1016/j.chemphys.2013.07.014.

34. Monajjemi, M.; Robert, W.J.; Boggs, J.E. NMR contour maps as a new parameter of carboxyl's OH groups in amino acids recognition: A reason of tRNA-amino acid conjugation. Chemical Physics 2014, 433, 1-11, https://doi.org/10.1016/j.chemphys.2014.01.017.

35. Monajjemi, M. Quantum investigation of non-bonded interaction between the B15N15 ring and BH2NBH2 (radical, cation, and anion) systems: a nano molecularmotor. Struct Chem 2012, 23, 551-580, https://dx.doi.org/10.1007/s11224-011-9895-8.

36. Monajjemi, M. Metal-doped graphene layers composed with boron nitride-graphene as an insulator: a nanocapacitor. Journal of Molecular Modeling 2014, 20, 2507, https://doi.org/10.1007/s00894-014-2507-y.

37. Monajjemi, M.; Heshmat, M.; Haeri, H.H.; Kaveh, F. Theoretical study of vitamin properties from combined QM-MM methods: Comparison of chemical shifts and energy. Russian Journal of Physical Chemistry 2006, 80, 1061-1068, https://doi.org/10.1134/S0036024406070119.

38. Monajjemi, M.; Honaparvar, B.; Khalili Hadad, B.; Ilkhani, A.; Mollaamin, F. Thermo-Chemical Investigation and NBO Analysis of Some anxileotic as Nano- Drugs. African journal of pharmacy and pharmacology 2010, 4, 521-529, https://doi.org/10.5897/AJPP.9000278.

39. Monajjemi, M., Najafpour, J., Mollaamin, F. (3,3) 4 Armchair carbon nanotube in connection with PNP and NPN junctions: Ab Initio and DFT-based studies. Fullerenes Nanotubes and Carbon Nanostructures 2013, 21, 213-232, https://doi.org/10.1080/1536383X.2011.597010.

40. Monajjemi, M., Jafari Azan, M., Mollaamin, F. Density functional theory study on B30N20 nanocage in structural properties and thermochemical outlook. Fullerenes Nanotubes and Carbon Nanostructures 2013, 21, 503-515, https://doi.org/10.1080/1536383X.2011.629762.

41. Monajjemi, M.; Ghiasi, R.; Ketabi, S.; Passdar, H.; Mollaamin, F. A Theoretical Study of Metal-Stabilised Rare Tautomers Stability: N4 Metalated Cytosine (M=Be2+, Mg2+, Ca2+, Sr2+ and Ba2+) in Gas Phase and Different. Journal of Chemical Research 2004, 1, 11-18, https://doi.org/10.3184/030823404323000648. 
42. Monajjemi, M., Baheri, H., Mollaamin, F. A percolation model for carbon nanotube-polymer composites using the Mandelbrot-Given. Journal of Structural Chemistry 2011, 52, 54-59, https://doi.org/10.1134/S0022476611010070.

43. Mahdavian, L., Monajjemi, M. Alcohol sensors based on SWNT as chemical sensors: Monte Carlo and Langevin dynamics simulation, Microelectronics Journal, 2010, 41(2-3), 142-149, 10.1016/ j.mejo. 2010.01.011.

44. Monajjemi, M., Heshmat, M., Haeri, H.H., Kaveh, F ,Theoretical study of vitamin properties from combined QM-MM methods: Comparison of chemical shifts and energy, Russian Journal of Physical Chemistry A, 2006, 80(7), 1061-1068, 10.1134/S0036024406070119.

45. Monajjemi, M., Afsharnezhad, S., Jaafari, M.R., ...Mollaamin, F., Monajemi, H, Investigation of energy and NMR isotropic shift on the internal rotation Barrier of $\Theta_{4}$ dihedral angle of the DLPC: A GIAO study, Chemistry, 2008, 17(1), 55-69

46. Mollaamin, F., Monajjemi, M. Harmonic linear combination and normal mode analysis of semiconductor nanotubes vibrations Journal of Computational and Theoretical Nanoscience, 2015, 12(6), pp. 1030-1039, $10.1166 /$ jctn.2015.3846

47. Yu, W.H.; Sing, S.L.; Chua, C.K.; Kuo, C.N.; Tian, X.L. Particle-reinforced metal matrix nanocomposites fabricated by selective laser melting: A state of the art review. Prog. Mater. Sci. 2019, 101, 330-379.

48. Chang, K.; Liang, E.; Huang, W.; Zhang, X.; Chen, Y.; Dong, J.; Zhang, R. Microstructural features and mechanical property in different directions of additive manufactured Ti6Al4V alloy. Mater. Lett. 2020, 267, $1-5$.

49. He, J.; Li, D.; Jiang, W.; Ke, L.; Qin, G.; Ye, Y.; Qin, Q.; Qui, D. The martensitic transformation and mechanical properties of Ti6Al4V prepared via selective laser melting. Materials 2019, 12, 321.

50. Sabban, R.; Bahl, S.; Chatterjee, K.; Suwan, S. Globularization using heat treatment in additively manufactured Ti6A14V. Acta Mater. 2019, 162, 239-254.

51. Algius, D.; Kourousis, K.I.; Wallbrink, C. A review of the as-built SLM Ti6Al4V mechanical properties towards achieving fatigue resistance designs. Metals 2018, 8, 1-24.

52. Exley, J.; Dickinson, M.; King, T.; et al: Comparison of cooling criteria with a cryogen spray and water/air spray. Proc SPIE, 1999, 3601, 130-140.

53. De Alencar, F.N.J.; Nonaka, C.F.W.; de Vasconcelos Catão, M.H.C. Effect of blue LED on the healing process of third-degree skin burns: Clinical and histological evaluation. Lasers Med. Sci. 2019, 34, 721-728.

54. Collins, N.J.; Hart, H.F.; Mills, K.A.G. OARSI year in review 2018: Rehabilitation and outcomes. Osteoarthr. Cartil. 2019, 27, 378-391.

55. Nowak, R.; Rekas, M.; Gospodarowicz, I.N.; Ali, M.J. Long-term outcomes of primary transcanalicular laser dacryocystorhinostomy. Graefe's Arch. Clin. Exp. Ophthalmol. 2021, 54, 1-6.

56. Arif, R.H.; Kareem, F.A.; Zardawi, F.M.; Al-Karadaghi, T.S. Efficacy of $980 \mathrm{~nm}$ diode laser and $2940 \mathrm{~nm}$ Er: YAG laser in gingival depigmentation: A comparative study. J. Cosmet. Dermatol. 2021, 20, 1684-1691.

57. Makdoumi, K.; Hedin, M.; Bäckman, A. Different photodynamic effects of blue light with and without riboflavin on methicillinresistant Staphylococcus aureus (MRSA) and human keratinocytes In Vitro. Lasers Med. Sci. 2019.

58. Abiri, A.; Bs, K.G.; Maducdoc, M.; Sahyouni, R.; Wang, M.B.; Kuan, E.C. Laser-Assisted Control of Epistaxis in Hereditary Hemorrhagic Telangiectasia: A Systematic Review. Lasers Surg. Med. 2020, 52, 293300.

59. Makdoumi, K.; Hedin, M.; Bäckman, A. Different photodynamic effects of blue light with and without riboflavin on methicillinresistant Staphylococcus aureus (MRSA) and human keratinocytes In Vitro. Lasers Med. Sci. 2019.

60. George, S.; Hamblin, M.R.; Abrahamse, H. Photobiomodulation-Induced Differentiation of Immortalized Adipose Stem Cells to Neuronal Cells. Lasers Surg. Med. 2020, 52, 1032-1040.

61. Hanke, A.; Fimmers, R.; Frentzen, M.; Meister, J. Quantitative determination of cut efficiency during soft tissue surgery using diode lasers in the wavelength range between 400 and $1500 \mathrm{~nm}$. Lasers Med. Sci. 2021, $1-15$.

62. Guerra, R.C.; de Luca, D.N.; Pereira, R.S.; Carvalho, P.H.A.; Homsi, N.; Radaic, P.; Pastore, G.P.; HochuliVieira, E. TMJ diode surgical laser approach in a contemporary treatment of tempomandibular joint pathologies. A technical note. Oral Surg. 2021, 14, 206-208.

63. Karkos, P.D.; Stavrakas, M.; Koskinas, I.; Markou, K.; Triaridis, S.; Constantinidis, J. 5 Years of Diode Laser "P" Technique for Bilateral Vocal Fold Immobility: A Technique That Improves Airway and Is Friendly to the Voice. Ear Nose Throat J. 2021, 100, 83S-86S. 
64. Espey, B.T.; Kielwein, K.; van der Ven, H.; Steger, K.; Allam, J.; Paradowska-Dogan, A.; van der Ven, K. Effects of Pulsed-Wave Photobiomodulation Therapy on Human Spermatozoa. Lasers Surg. Med. 2021.

65. Xiang, A.; Deng, H.; Cheng, K.; Liu, H.; Lin, L.; Qu, X.; Liu, S.; Shen, X. Laser photobiomodulation for cartilage defect in animal models of knee osteoarthritis: A systematic review and meta-analysis. Lasers Med. Sci. 2020, 35, 789-796.

66. Campiche, R.; Curpen, S.J.; Lutchmanen-Kolanthan, V.; Gougeon, S.; Cherel, M.; Laurent, G.; Gempeler, M.; Schuetz, R. Pigmentation effects of blue light irradiation on skin and how to protect against them. Int. J. Cosmet. Sci. 2020, 42, 399-406.

67. Szyma 'nski, Ł.; J، ederka, K.; Cios, A.; Ciepelak, M.; Lewicka, A.; Stankiewicz, W.; Lewicki, S. A simple method for the production of human skin equivalent in 3D, multi-cell culture. Int. J. Mol. Sci. 2020, $21,4644$. 